\title{
A SURVEY OF GLOBULAR CLUSTER VELOCITY DISPERSIONS
}

\author{
Carlton Pryor \\ University of Victoria and Vanderbilt University \\ Robert D. McClure*, J. M. Fletcher and James E. Hesser \\ Dominion Astrophysical Observatory \\ Hertzberg Institute of Astrophysics
}

We have used the Dominion Astrophysical Observatory radial velocity' spectrometer on the $3.6 \mathrm{~m}$ Canada-France-Hawaii telescope to obtain radial velocities, accurate to $\sim 0.8 \mathrm{~km} / \mathrm{s}$, for $\sim 20$ stars in each of nine globular clusters. The stars are generally within three core radii of the cluster center. The cluster names and metallicities (the latter are averages of values in Zinn and West (1984), Pilachowski (1984), and Webbink (1985)) are given in Table I. This sample includes two clusters with cusps in their surface brightness profiles: NGC 6624 and 6681.

We fit multi-component King models with isotropic velocity dispersions to our velocity data and surface brightness profiles taken from the literature. The velocity data are fitted using Gunn and Griffin's (1979) maximum likelihood technique, which removes the contribution of the measurement uncertainty. The surface brightness profiles are fitted using least squares. Uncertainties in the cluster parameters are determined by fitting many simulated data sets. We do not include the large uncertainty in the cluster distances. The distances are calculated assuming that the $R R$ Lyrae variables have $M_{V}=+0.6$. To be acceptable, a model must be a good fit to the surface brightness profile and the mass-to-light ratio $(M / L)$ from the fit must agree with the $M / L$ of the mass function used in the model.

Table I gives our best estimates of the absolute visual magnitude $\left(\mathrm{MV}_{\mathrm{V}}\right)$, the concentration parameter (c), the mean cluster velocity $(<v>)$, the central projected velocity dispersion $\left(\mathrm{PV}_{\mathrm{o}}\right)$, and the global and central $M / L_{\mathrm{V}}$ 's. We define $\left(M / L_{\mathrm{V}}\right)_{0}$ to be the ratio of the central mass and luminosity surface densities. These values are based on fits of multi-component models with mass function exponents, $x$, of $0.67,1.35$, and $2.00\left(d N \propto m^{-x} d \log (m)\right)$. Often, all three exponents produce acceptable fits. If this results in a range of values for a parameter that is larger than the uncertainties in the individual fits, the range is given in the table.

Mass functions with exponents of 1.35 and 2.00 yield acceptable models for all of the clusters in our sample. An $x$ of 0.67 is acceptable for all of the clusters

* Visiting Astronomer, Canada-France-Hawaii Telescope, operated by the National Research Council of Canada, the Centre National de la Recherche Scientifique of France, and the University of Hawaii. 
except NGC 6171, 6218, and 6624. We have also tried models with $x=0.0$ and they are not consistent for the clusters in our sample. The theoretical $M / L_{V}(3.2)$ is always at least $2 \sigma$ larger than the value derived from the fit. This arises because the shallow mass function predicts a large number of white dwarfs with masses equalling or exceeding that of the giants (we assume that stars with initial masses less than $8 M_{\odot}$ make white dwarfs). We have fitted models with an anisotropy radius of $10 r_{c}$ to NGC 6624 and 6681 and, for these clusters at least, the acceptable $x$ values do not depend strongly on the assumption of isotropy.

Table I.

Results From Fitting Isotropic Multi-Component King Models

\begin{tabular}{|lcccccccc|}
\hline NGC $[\mathrm{Fe} / \mathrm{H}]$ & $\mathrm{MV}_{\mathrm{V}}$ & $\mathrm{c}$ & $\begin{array}{c}\# \text { of } \\
\text { stars }\end{array}$ & $\begin{array}{c}\langle v\rangle \\
(\mathrm{km} / \mathrm{s})\end{array}$ & $\begin{array}{c}\mathrm{PV} \\
(\mathrm{km} / \mathrm{s})\end{array}$ & $\begin{array}{c}M / L_{\mathrm{V}} \\
\left(M_{\odot} / L_{\mathrm{O}}\right)\end{array}$ & $\begin{array}{c}\left(M / L_{\mathrm{V}}\right)_{\mathrm{o}} \\
\left(L_{\mathrm{O}}\right)\end{array}$ \\
\hline 288 & -1.2 & $-6.71 \pm .09$ & 1.0 & 26 & $-45.9 \pm 0.6$ & $2.9 \pm 0.5$ & $2.6 \pm 0.8$ & $2.0 \pm 0.6$ \\
4147 & -1.8 & $-6.00 \pm .07$ & 1.7 & 9 & $183.2 \pm 0.9$ & $2.8 \pm 0.9$ & $2.8 \pm 1.7$ & $1.7 \pm 0.9$ \\
5466 & -1.9 & $-6.85 \pm .08$ & 1.0 & 20 & $107.7 \pm 0.4$ & $2.0 \pm 0.4$ & $1.7 \pm 0.6$ & $1.3 \pm 0.5$ \\
6171 & -0.9 & $-6.87 \pm .15$ & 1.7 & 18 & $-32.8 \pm 0.7$ & $2.9 \pm 0.6$ & $2.4 \pm 0.9$ & $1.0 \pm 0.4$ \\
6218 & -1.6 & $-7.08 \pm .11$ & 1.3 & 20 & $-45.0 \pm 0.8$ & $3.7 \pm 0.7$ & $1.9 \pm 0.6$ & $1.1 \pm 0.4$ \\
6624 & -0.7 & $-7.53 \pm .14$ & $2.3:$ & 19 & $54.1 \pm 1$ & $5.4 \pm 1$ & $1.6-3.8$ & $1.2 \pm 0.5$ \\
6626 & -1.5 & $-8.19 \pm .13$ & 1.8 & 19 & $15.9 \pm 2$ & $8.9 \pm 1.5$ & $1.5-5$ & $1.6 \pm 0.6$ \\
6681 & -1.2 & $-7.23 \pm .15$ & $2.2:$ & 18 & $218.8 \pm 1$ & $5.2 \pm 1$ & $1.5-5$ & $2.1 \pm 0.9$ \\
6809 & -1.6 & $-7.41 \pm .06$ & 0.9 & 20 & $176.8 \pm 0.9$ & $4.2 \pm 0.7$ & $1.7 \pm 0.6$ & $1.4 \pm 0.4$ \\
\hline
\end{tabular}

That none of our clusters are consistent with a flat mass function is surprising since McClure et al. (1986) found several clusters whose main sequence luminosity functions yielded $x \simeq 0.0(x<0.0$ when mass segregation corrections are applied, see Pryor, McClure, and Smith (1986)). NGC 6624 appears to have a steeper mass function than the correlation of $x$ with metallicity found by McClure et al. would suggest. However, a main sequence luminosity function has not been measured.

Although the King models that we use underestimate the luminosity at the center of a cusp cluster, they also underestimate the mass there. In agreement with this, our central $M / L$ 's show little dependence on the concentration of the model used to derive them. The low central $M / L$ of NGC 6624 suggests that giants make the dominant contribution to the density in its inner regions. This is consistent with the giants showing an $r^{-1}$ profile (Djorgovski and King, 1984), since they would be the heaviest important component. On the other hand, NGC 6681 has the largest central $M / L$ in our sample. Based on this sample of two, cusp clusters do not seem to have $M / L$ 's significantly different from those of the cluster population as a whole.

\section{REFERENCES}

Djorgovski, S. and King, I. R. 1984 Astrophys. J. Letters 277, 249. Gunn, J. E. and Griffin, R. F. 1979 Astron. J. 84, 752.

McClure, R. D., VandenBerg, D. A., Smith, G. H., Fahlman, G. G., Richer H. B., Hesser, J. E., Harris, W. E., Stetson, P. B. and Bell, R. A. 1986 Astrophys. J. Letters 307, 247.

Pilachowski, C. A. 1984 Astrophys. J. 281, 614.

Pryor, C., McClure, R. D. and Smith, G. H. 1986 Astron. J. 92, in press.

Webbink, R. F. 1985 in IAU Symposium No. 113, Dynamics of Star

Clusters, J. Goodman and P. Hut, eds., Reide1, Dordrecht, p. 541.

Zinn, R. and West, M. J. 1984 Astrophys. J. Suppl. 55, 45. 